\title{
Fatal Strongyloidiasis in Three Kidney Recipients in Kuwait
}

\author{
Eiman M. Mokaddas ${ }^{a, b}$ Shama Shati ${ }^{b}$ Aneesa Abdulla ${ }^{b}$ \\ Narayanan R. Nampooric Jamshaid lqbal ${ }^{\mathrm{a}}$ Prasad M. Nair ${ }^{c}$ Tareq Said $^{\mathrm{c}}$ \\ Medhat Abdulhalim ${ }^{c}$ Parsotam R. Hira ${ }^{a}$ \\ ${ }^{a}$ Department of Microbiology, Faculty of Medicine, Kuwait University, ${ }^{b}$ Laboratory Department and \\ 'Hamed Al Essa Organ Transplant Center, Ibn Sina Hospital, Kuwait
}

\section{Key Words}

Strongyloidiasis $\cdot$ Kidney transplant $\cdot$ Immunocompromised

\begin{abstract}
Objective: It was the aim of this study to report 3 rare fatal cases of strongyloidiasis in Kuwaiti renal transplant patients.

Clinical Presentation and Intervention: All 3 cases received allografts from cadaveric donors of Asian origin, the first 2 from an Indian (transplanted on the same day) and the third from a Bangladeshi. In all 3 cases, Strongyloides stercoralis larvae were first isolated from bronchoalveolar lavage. All 3 patients were on immunosuppressive therapy which included prednisolone, thereby leading to the hyperinfection syndrome. All patients presented with gastrointestinal symptoms (abdominal pain, vomiting, diarrhea, constipation and paralytic ileus), as well as pulmonary symptoms (cough, dyspnea and blood-stained sputum). Albendazole $800 \mathrm{mg}$ twice daily orally was started. Cyclosporine A was started after discontinuing prograf. The patients continued to deteriorate with a fall in blood pressure and platelets. All 3 patients died from adult respiratory distress syndrome following hyperinfection with S. stercoralis. Conclusion: Hyperinfection with S. stercoralis is a rare but preventable complication of immunosuppressive therapy. A high index of suspicion is required
\end{abstract}

for the diagnosis of this infection. Persistent examination of sputum, bronchial washings and upper intestinal aspirates should be done as part of surveillance following cadaveric renal transplantation. Adult respiratory distress syndrome is indeed a red flag in patients who are on steroids, not on cyclosporine and receiving a kidney from donors in endemic countries of S. stercoralis.

Copyright $\odot 2009$ S. Karger AG, Basel

\section{Introduction}

Strongyloides stercoralis, an intestinal nematode of man with worldwide distribution, may cause symptoms in the infected individual depending on the worm load, but can be life threatening in immunocompromised hosts. S. stercoralis infection initiates by the filariform larvae which develop through the direct cycle, indirect cycle and autoinfection, both exo- and endoautoinfection. The adult female parasitizing the human small intestine lays eggs that hatch into larvae which are passed in the stool. Strongyloidiasis is often considered a disease of tropical and subtropical areas. In immunocompromised individuals, the infection is characterized by extensive larval invasion of the gastrointestinal and respira- 
tory tracts and is referred to as the hyperinfection syndrome. Invasion of multiple organs may also occur and is referred to as disseminated strongyloidiasis [1].

Complications such as paralytic ileus, malabsorption, gastrointestinal hemorrhage, Gram-negative bacteremia, meningitis and severe pneumonia with adult respiratory distress syndrome (ARDS) have also been reported [2]. Although strongyloidiasis is not commonly recognized as a complication of renal transplantation, several cases have been reported worldwide [3]. Treatment options are limited to oral formulations with no available parenteral chemotherapy. Here, we report 3 cases of fatal strongyloidiasis in renal transplant patients in Kuwait.

\section{Case Reports}

\section{Case 1}

A 52-year-old male Kuwaiti underwent a cadaveric renal transplant from an Indian donor following diabetic nephropathy and renal failure. He was on immunosuppressive therapy which included prograf ( $6 \mathrm{mg}$ twice daily), cellcept ( $1 \mathrm{~g}$ twice daily) and prednisolone (20 mg once daily). Forty-seven days after transplant, he presented at the outpatient clinic with abdominal pain and intractable vomiting of 1 day duration. His abdomen was tender and had signs of dehydration. He was febrile $\left(39^{\circ} \mathrm{C}\right)$. His blood count did not show eosinophilia. A direct smear of the stool did not reveal any ova or parasites. The next day, he developed diarrhea, became disoriented and confused. Blood culture revealed Escherichia coli septicemia. The patient was started on piperacillin/tazobactam parenterally $4.5 \mathrm{~g} / 8$ hourly. He continued to deteriorate and there was a drop in oxygen saturation, blood pressure $(90 / 60 \mathrm{~mm} \mathrm{Hg})$ and platelets $\left(93 \times 10^{9} / \mathrm{l}\right)$. His blood urea was raised $(77.8 \mathrm{mmol} / \mathrm{l})$ and he went into anuria. Chest X-ray showed signs of ARDS. He was admitted into the intensive care unit and intubated. Bronchoalveolar lavage was done 55 days after transplant and revealed S. stercoralis rhabditiform larvae on direct microscopy $(5-7 / \mathrm{hpf})$. The larvae were actively motile. Oral albendazole $800 \mathrm{mg}$ twice daily was started via the nasogastric tube. The next day, nasogastric tube aspirates and endotracheal tube secretions also showed actively motile S. stercoralis larvae. Albendazole $400 \mathrm{mg}$ twice daily was started as retention enema. Prograf was discontinued and cyclosporine A $100 \mathrm{mg}$ twice daily was started. The dose of prednisolone was tapered. Ivermectin $12 \mathrm{mg}$ twice daily was started via the nasogastric tube. There was a further drop in saturation, blood pressure was not recordable and in spite of all the supportive measures, the patient expired 66 days after transplant.

\section{Case 2}

A 43-year-old female Kuwaiti received a cadaveric kidney from the same Indian donor as the first patient on the same day for end-stage renal disease. She was on immunosuppressive therapy which included prograf ( $3 \mathrm{mg}$ twice daily), cellcept (1 g twice daily) and prednisolone (15 mg once daily). She presented with vomiting, diarrhea, abdominal pain and difficulty in breathing,
90 days after transplant. She was afebrile and her blood count showed no eosinophilia. Her serum creatinine was raised (147 $\mu \mathrm{mol} / \mathrm{l})$. Direct stool smears showed no ova or parasites. Chest $\mathrm{X}$-ray revealed a right basal pneumonia. Meropenem was started intravenously. A bronchoalveolar lavage was done 102 days after transplant which revealed the presence of S. stercoralis larvae. Albendazole $800 \mathrm{mg}$ twice daily orally was started. Cyclosporine A was started after discontinuing prograf. The patient continued to deteriorate with a fall in blood pressure and platelets. The liver enzymes were raised and she developed deep jaundice. Total bilirubin was $163 \mu \mathrm{mol} / \mathrm{l}$ (normal 0-17) and direct bilirubin was 117 $\mu \mathrm{mol} / \mathrm{l}$ (normal 0-5). There was a further fall in blood pressure, platelets and saturation. The patient went into anuria and expired 106 days after transplant.

\section{Case 3}

A 43-year-old female Kuwaiti underwent a cadaveric renal transplant from a Bangladeshi for chronic renal failure. She was on prograf (4 mg twice daily), cellcept (1 g twice daily) and prednisolone (15 mg once daily). She presented with abdominal distension, nausea, constipation and rash around the umbilicus 91 days after transplant. She was febrile $\left(38^{\circ} \mathrm{C}\right)$ and had a cough with blood-stained sputum. Chest X-ray revealed bilateral fluffy shadows. Bronchoalveolar lavage done 93 days after transplant showed S. stercoralis larvae. Albendazole $400 \mathrm{mg}$ twice daily was started through the nasogastric tube but her condition deteriorated rapidly and she expired 95 days after transplant.

\section{Discussion}

In a study of stongyloidiasis in Kuwait, the risk groups vulnerable to strongyloidiasis in a nonendemic country were elaborated but no kidney transplant patients were reported [4]. Opportunistic infection with S. stercoralis is a potentially preventable cause of disease and death in renal transplant patients provided there is a high index of clinical suspicion. Patients usually present with pulmonary and gastrointestinal symptoms as seen in our cases $[1,5]$.

All 3 patients were on immunosuppressive therapy which included prednisolone, thereby leading to the hyperinfection syndrome. Prednisolone acts by accelerating the rate of ecdysis of the rhabditiform larvae. It has been reported that major morbidity and death occurs 1-3 months after transplantation [2]. In our cases, the first patient presented 47 days after transplantation and the second and third cases after approximately 3 months. It may well be asked why no previous reports of stongyloidiasis were documented despite an active renal transplant program in Kuwait. A possible reason for no previous reports of stongyloidiasis documented in Kuwait may be the use of cyclosporine $\mathrm{A}$ in all renal transplant patients which is known to have antihelminthic activity [6]. 
None of the 3 cases received cyclosporine A as post-transplant management.

All patients presented with gastrointestinal and pulmonary symptoms. ARDS was a common manifestation in all 3 cases. It proved to be fatal and is consistent with reports by Thompson and Berger [2].

Delay in the diagnosis of strongyloidiasis has been associated with increased morbidity and mortality, as in our cases. Other factors that increase the mortality rate are continued steroid therapy, persisting parasitism, disseminated disease and bacteremia. Absence of eosinophilia, as in all our cases, correlated with the poor prognosis and high mortality $[1,3]$.

In all our 3 cases, the diagnosis of strongyloidiasis was delayed because there was no history of travel or stay in the endemic areas. Since Kuwait is not an endemic area, patients and donors are not screened for strongyloidiasis before transplantation. The cadaveric kidneys were from Asian donors. Stool examination of patients did not reveal any ova or parasite. A single stool examination is only $50 \%$ sensitive, a minimum of 4 stool samples should be examined to rule out infection in suspected cases. Many of the early clinical manifestations were attributed to the immunosuppressive state of renal transplant patients. Fever, cough and hemoptysis in the presence of roentgenographic infiltrates suggested other etiologies. Bronchoalveolar lavage specimens gave the highest yield, as seen in the 3 cases. Serologic testing is now widely available and is sensitive although not very specific. Infections with filarial or round worm can lead to false positives.

Prompt and rapid institution of therapy is one of the most important determinants of outcome of the disease. There are several obstacles in the management of strongyloidiasis: (1) therapeutic failures suggest that an effective and adequate antihelminthic therapy is not well established, particularly in the immunocompromised hosts, (2) decreased cell-mediated immunity due to immunosuppression probably diminishes the effect of medical therapy, and (3) paralytic ileus may lead to poor absorption of oral antihelminthic agents, as was observed in our patients $[7,8]$.

All antihelminthics with activity against $S$. stercoralis are oral agents. Effective parenteral drug therapy is not currently available. Cyclosporine $\mathrm{A}$ is believed to be a potent inhibitor of a range of parasites and acts on $\mathrm{T}$ lymphocytes as complexes with their binding proteins, cyclophilins [9]. Unfortunately, none of our 3 patients were on cyclosporine A. Thiabendazole is the recommended drug of choice in immunosuppressed patients.
Rectal administration of thiabendazole in patients with strongyloides hyperinfection syndrome has proved to be effective. However, this drug was not available to treat the 3 cases in Kuwait. Ivermectin is also an effective drug in the treatment of disseminated strongyloidiasis in the immunocompromised. Ivermectin enemas proved to be effective, particularly in those with paralytic ileus. The first 2 cases were treated with ivermectin which was available only during the later course of the illness.

Cases 1 and 2 received a kidney from the same cadaver donor of Indian origin who died of a road traffic accident. The cadaver donor was the likely source of infection as India is a known endemic area and many such patients may not show any clinical signs of infection. However, it could not be confirmed due to the nonavailability of his medical record. The larvae usually survive the relatively short cold ischemia time to which kidneys are subjected before being transplanted and could have been the source of infection in the recipient [6].

Another likely mode of transmission, faeco-oral [6], although rare, was also investigated. Screening prior to transplantation of both the donors and the recipients allows early detection and treatment of infection. We suggest that donors from endemic countries should be given a treatment dose of ivermectin before harvesting the kidney.

\section{Conclusion}

Hyperinfection with S. stercoralis is a rare but preventable complication of immunosuppressive therapy. A high index of suspicion is required for the diagnosis of this infection. Emphasis should be laid on routine examination of all donors and recipients for ova, cysts and parasites. Persistent examination of sputum should be done as part of surveillance following cadaveric renal transplantation. Clinicians with an awareness of the possibility of hyperinfection are better equipped to diagnose, treat or altogether prevent the fatal consequences of this lethal nematode. ARDS is indeed a red flag in patients who are on steroids, not on cyclosporine and receiving a kidney from donors in endemic countries. 


\section{References}

- I Igra-Siegman Y, Kapila R, Sen P, Kaminski ZC, Louria DB: Syndrome of hyperinfection with Strongyloides stercoralis. Rev Infect Dis 1981;3:397-407.

$>2$ Thompson JR, Berger R: Fatal adult respiratory distress syndrome following successful treatment of pulmonary strongyloidiasis. Chest 1991;99:772-774.

3 Morgan JS, Schaffner W, Stone WJ: Opportunistic strongyloidiasis in renal transplant patients. Transplantation 1986;42:518-524.
-4 Hira PR, Al-Ali F, Shweiki HM, Abdella NA, Johny M, Francis I, Iqbal J, Thompson R, Neva F: Strongyloidiasis: challenges in diagnosis and management in non-endemic Kuwait. Ann Trop Med Parasitol 2004;98:261270.

5 Liepman M: Disseminated Strongyloides stercoralis: a complication of immunosupression. JAMA 1975;231:387-388.

6 Palau LA, Pankey GA: Strongyloides hyperinfection in a renal transplant recipient receiving cyclosporine: possible Strongyloides stercoralis transmission by kidney transplant. Am J Trop Med Hyg 1997;57:413415.
7 Nonaka D, Takaki K, Tanaka M, Umeno M, Takeda T, Yoshida M, Haraguch Y, Okada K, Sawai Y: Paralytic ileus due to strongyloidiasis: case report and review of literature. Am J Trop Med Hyg 1998;59:535-538.

$>8$ Porto AF, Neva FA, Bittencourt H, Lisboa W, Thompson R, Alcantara L, Carvalho EM: HTLV-1 decreases Th 2 type of immune response in patients with strongyloidiasis. Parasite Immunol 2001;23:503-507.

9 Schad GA: Cyclosporine may eliminate the threat of overwhelming strongyloidiasis in immunosuppressed patients. J Infect Dis 1986;153:178. 